\title{
ERRATUM
}

Published online: 2012

(C) The Journal of Nutrition, Health \& Aging 2012

Erratum to: The Journal of Nutrition, Health \& Aging

DOI 10.1007/s12603-012-0072-6

\section{Erratum to: Glomerular filtration rate estimated by Cockcroft-Gault formula better predicts anti-Xa levels than Modification of the Diet in Renal Disease equation in older patients with prophylactic enoxaparin}

B. Dufour ${ }^{1}$, M. Toussaint-Hacquard ${ }^{2}$, A. Kearney-Schwartz ${ }^{1,3}$, M.D.P. Manckoundia ${ }^{4}$, M.-C. Laurain ${ }^{1}$, L. Joly ${ }^{1}$, J. Deibener ${ }^{1}$, D. Wahl ${ }^{5,6}$, T. Lecompte ${ }^{2,6}$, A. Benetos ${ }^{1,3,6}$, C. Perret-Guillaume ${ }^{1,3,7}$

1. Geriatric Unit, Hôpital de Brabois, CHU Nancy, France; 2. Haematology Laboratory, Hôpital de Brabois, CHU Nancy, France; 3. Inserm - Clinical Investigation Center, CIC9501, Institut Lorrain du Coeur et des Vaisseaux Louis Mathieu, Nancy, France; 4. Department of Internal Medicine and Geriatrics, University Hospital, Dijon, France; 5. Department of vascular medicine, Hôpital de Brabois, CHU Nancy, France; 6. INSERM U961, Faculté de Médecine, Vandoeuvre-les-Nancy, France; 7. Nancy-Université, Université Paul Verlaine Metz, Université Paris Descartes, EA 4360 Apemac, Nancy, France. Corresponding author : Christine Perret-Guillaume, Geriatric Unit, Hôpital de Brabois, CHU Nancy, Nancy, France, Phone number 333383153322, fax number 333383157668, email : c.perret-guillaume@ chu-nancy.fr

The already published version of the article "Glomerular filtration rate estimated by Cockcroft-Gault formula better predicts anti-Xa levels than Modification of the Diet in Renal Disease equation in older patients with prophylactic enoxaparin" contains a spelling error in the authors list. M.D.P. Manckoundia should be spelled P. Manckoundia. 